\title{
Genetic signatures of ecological diversity along an urbanization gradient
}

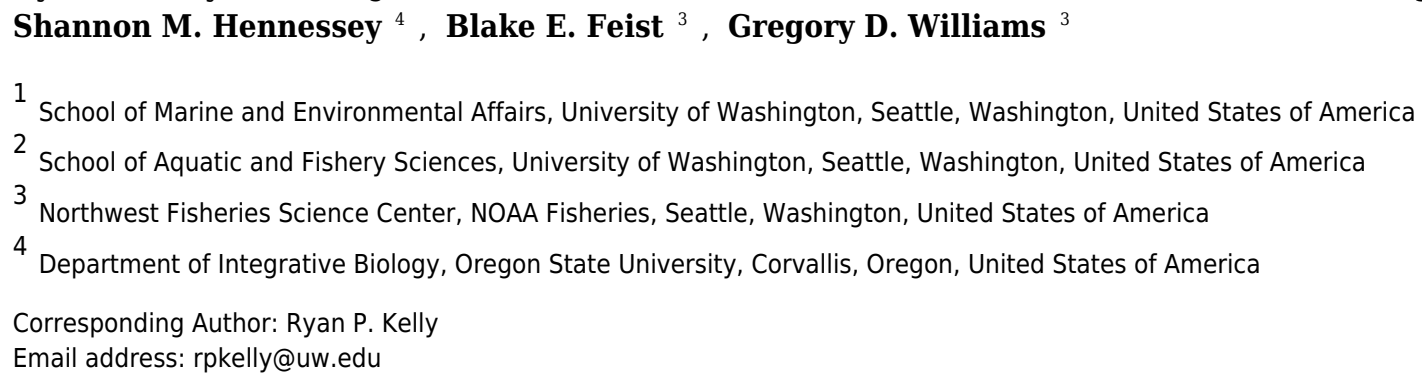

Despite decades of work in environmental science and ecology, estimating human influences on ecosystems remains challenging. This is partly due to complex chains of causation among ecosystem elements, exacerbated by the difficulty of collecting biological data at sufficient spatial, temporal, and taxonomic scales. Here, we demonstrate the utility of environmental DNA (eDNA) for quantifying associations between human land use and changes in an adjacent ecosystem. We analyze metazoan eDNA sequences from water sampled in nearshore marine eelgrass communities and assess the relationship between these ecological communities and the degree of urbanization in the surrounding watershed. Counter to conventional wisdom, we find strongly increasing richness and decreasing beta diversity with greater urbanization, and similar trends in the diversity of life histories with urbanization. We also find evidence that urbanization influences nearshore communities at local (hundreds of meters) rather than regional (tens of $\mathrm{km}$ ) scales. Given that different survey methods sample different components of an ecosystem, we then discuss the advantages of eDNA-which we use here to detect hundreds of taxa simultaneously-as a complement to traditional ecological sampling, particularly in the context of broad ecological assessments where exhaustive manual sampling is impractical. Genetic data are a powerful means of uncovering human-ecosystem interactions that might otherwise remain hidden; nevertheless, no sampling method reveals the whole of a biological community. 


\title{
Genetic Signatures of Ecological Diversity Along an Urbanization Gradient
}

\author{
Ryan P. Kelly*1, James L. O'Donnell'1, Natalie C. Lowell², Andrew O. \\ Shelton $^{3}$, Jameal F. Samhouri ${ }^{3}$, Shannon M. Hennessey ${ }^{4}$, Blake E. Feist ${ }^{3}$, \\ Gregory D. Williams ${ }^{3}$
${ }^{I}$ School of Marine and Environmental Affairs, University of Washington, 3707 Brooklyn Ave NE, Seattle, Washington 98105, USA
${ }^{2}$ School of Aquatic and Fishery Sciences, University of Washington, 1122 NE Boat St, Seattle, Washington 98105, USA USA \\ ${ }^{3}$ NOAA Fisheries, Northwest Fisheries Science Center, 2725 Montlake Blvd E, Seattle, Washington 98112, USA \\ ${ }^{4}$ Department of Integrative Biology, Oregon State University, 3029 Cordley Hall, Corvallis, Oregon 97331, \\ *Corresponding author: rpkelly@uw.edu
}

21 
22

23

24

25

26

27

28

29

30

31

32

33

34

35

36

37

38

39

40

41

42

43

44

45

46

47

48

49

50

51

52

53

54

55

56

57

58

59

60

61

62

63

64

65

66

67

\section{Introduction}

An enduring question of environmental science and ecology is how to measure the effects of human activities on nearby biological communities and ecosystems. While in some cases such impacts are so obvious that in-depth sampling is unnecessary to reveal them-such as paving over a wetland or clear-cutting a rainforest - many human activities are likely to have more subtle effects on the surrounding system. More adequately measuring human impacts is a core challenge as human demands on natural resources continue to grow; such measurement is a prerequisite for identifying sustainable development pathways.

The difficulty of surveying ecological communities generally results in a depth-vs.-breadth (i.e., specificity, (Rice \& Rochet, 2005)) tradeoff in sampling strategy. For example, one might comprehensively survey indicator taxa with the idea that they reflect larger changes to the ecological community (Niemi \& McDonald, 2004), or instead build limited data from many taxa into multimetric indices in an attempt to reflect some more holistic sense of ecosystem integrity (Karr, 1981; Weisberg et al., 1997). Environmental DNA (eDNA) could substantially improve upon existing survey methods by mitigating this tradeoff (Karr, 1981; Weisberg et al., 1997) by providing in-depth views of ecosystems at levels of effort comparable to traditional sampling. Indeed, microbial ecology has used these same core techniques for a decade or more (Tyson et al., 2004; Venter et al., 2004; Yutin et al., 2007). Sequencing the diagnostic traces of genetic material in environmental samples makes it possible to detect hundreds or thousands of animals, plants, and other organisms from target habitats on ecological time scales of hours to days (Thomsen et al., 2012; Turner et al., 2014). Yet although the rapid rise of eDNA as a tool for ecological studies has featured methodological leaps and assessments of performance (Thomsen et al., 2012; Ficetola et al., 2014; Thomsen \& Willerslev, 2015; Evans et al., 2016), the value that community-level eDNA methods add to traditional ecological sampling is just beginning to be apparent.

Measuring the influence of urban development on surrounding ecosystems is one application for which the broad scope of eDNA sampling may be particularly useful, in part because of the many pathways through which correlates of urbanization are likely to influence nearby ecological communities. Accordingly, it may be difficult to identify diffuse urban impacts using traditional ecological sampling alone, a particularly pressing problem as coastal urbanization increases globally (Neumann et al., 2015). For example, in Puget Sound, Washington, USA, as in many coastal areas, homeowners modify or harden their shorelines with concrete or other materials to protect their properties from erosion(Scyphers, Picou \& Powers, 2015). Permitting for shoreline armoring can create conflicts between individual property rights and the communal benefits that arise from unarmored shoreline, which include storm- and flood mitigation, habitat, waterline access, and other services. Laborious manual sampling has documented some shifts in ecology as a result of shoreline armoring (Heerhartz et al., 2014), but the ability to detect the ecosystem effects of any stressor depends strongly upon the choice of taxa sampled. Making such informed decisions about the scope of sampling is a general problem in ecology and environmental sciences.

We assessed the effects of upland watershed urbanization on nearshore estuarine eelgrass (Zostera marina) communities in Puget Sound, Washington, USA using eDNA sampling at four pairs of moreand less-urban sites (Fig. 1). Puget Sound has experienced rapid urbanization over the past century, its human population increasing nearly six-fold since 1920 (Minnesota Population Center, 2011), and nearly 4 million people live within $20 \mathrm{~km}$ of its shore (Bright et al., 2012). Although preserving 
68 biogenic eelgrass habitat is now a policy priority for state and federal agencies (Puget Sound

69 Partnership, 2011; US Army Corps of Engineers, 2012), the effect of such urbanization on eelgrass-

70 associated fauna has been difficult to characterize with traditional sampling techniques (e.g., (Blake,

71 Duffy \& Richardson, 2014) in Chesapeake Bay). As such, the steep urbanization gradient of Puget

72 Sound makes a compelling setting for evaluating eDNA as a means of detecting ecological differences associated with human development. Here, we report significant changes in community composition, diversity, and life-history composition associated with upland urbanization, as measured by the genetic signatures of animals detected in the water.

\section{Methods}

We selected 8 sites in nearshore eelgrass habitats adjacent to watersheds along a gradient of urbanization in Puget Sound, Washington, USA (Fig. 1). We employed a paired study design, in which each more-urbanized site had a companion less-urbanized site at approximately the same latitude (Fig. 1), controlling for well-known geographic, oceanographic, and ecological gradients within the Sound (Dethier, 2010). These were a subset of the sampling sites described in (Samhouri et al.), and included Big Gulch Creek (BG), Clearwater Casino (CC), Clinton-Whidbey (CW), Manchester (MA), Pipers Creek (PC), Redondo Beach Cold Creek (RB), Sinclair Inlet (SI), and Shingle Mill Creek (SM). Further site details and coordinates are given in (Samhouri et al.).

\section{Environmental Setting}

We chose sites on the basis of watershed-scale patterns of urbanization as further described in (Samhouri et al.). All watershed basins were less than 1,000 ha, and contained perennial streams (Puget Sound Nearshore Ecosystem Restoration Project, 2010). We used three different geospatial data layers that captured various aspects of terrestrial urbanization-imperviousness (Fry et al., 2011), roadways (OpenStreetMap, 2013), and percent developed land cover (NOAA, 2013) - as well as percent shoreline armoring (Puget Sound Nearshore Ecosystem Restoration Project, 2010), to characterize urbanization at each site. Each of these individual metrics positively covaried and ordination techniques did not result in an index that was significantly more useful than any one urbanization variable alone. We therefore simply used imperviousness (the area-weighted mean percent cover of impervious surface) here as a proxy for human population and other urbanizationrelated parameters. This layer represents highly- to completely impermeable surfaces such as building roofs, concrete or asphalt roads and parking lots, concrete, asphalt or brick sidewalks, pedestrian walkways, and malls. We used Environmental Systems Research Institute's (Esri) ArcGIS software suite (v. 10.1) for all spatial analyses. Within site pairs, more-urban sites had higher values of imperviousness than their less-urban counterparts. Other environmental variables such as seasurface temperature (mean, max, SD) and salinity did not systematically vary with urbanization across our sites.

\section{eDNA Collection, Extraction, and Sequencing}

In July 2014, we collected 1-liter water samples for eDNA analysis at each of three transects within each site, and kept these on ice until they could be processed in the lab (within hours of collection). We filtered samples onto cellulose acetate filters $(47 \mathrm{~mm}$ diameter; $0.45 \mathrm{um}$ pore size) under vacuum pressure, and preserved the filter at room temperature in Longmire's buffer following Renshaw et al. (Renshaw et al., 2015). Deionized water (1-liter) served as a negative control for filtering. We extracted total DNA from the filters using the phenol:chloroform:isoamyl alcohol protocol in (Renshaw et al., 2015), resuspended the eluate in $200 \mathrm{uL}$ water, and used $1 \mathrm{uL}$ of diluted DNA extract 
115 (1:100, diluted to reduce amplification inhibition) as template for PCR. Total DNA recovered from

116 samples (quantified using a Qubit fluorometer) was uncorrelated with site urbanization, indicating

117 our results were not due to an accumulation of eDNA in environments near urban sites. See

118 Supplementary Methods for additional sampling details.

119

120

121

122

123

124

125

126

127

128

We designed a novel set of primers using ecoPrimers (Riaz et al., 2011) to amplify approximately 114-140bp of mitochondrial 16S DNA from metazoans exclusively. These primers effectively amplify most major animal phyla —including representatives from Chordata, Arthropoda, Mollusca, Echinodermata, Nemertea, and others-while excluding non-metazoans entirely. Their sequences are as follows (5' to 3'): 16s_Metazoa_fwd AGTTACYYTAGGGATAACAGCG; 16s_Metazoa_rev CCGGTCTGAACTCAGATCAYGT.

129

130

131

132

133

134

135

136

137

138

We generated amplicons using a two-step PCR procedure, described in (O’Donnell James L. et al., 2016), to avoid the taxon-specific amplification bias that results from the use of differentially indexed PCR primers (commonly used to include multiple samples onto the same high-throughput sequencing run to minimize costs). The specific PCR protocol is included in Supplementary Methods.

Each of the 24 environmental samples ( 3 samples/site, 8 sites) was amplified in a total of four PCR reactions, twice with each of two distinct indexed primer sets (see Supplementary Methods for indexing details), for a total of $24 \times 4=96$ individual sets of amplicons for sequencing. All but one of the environmental samples (from site $\mathrm{CW}$ ) was sequenced successfully. We also sequenced four positive (Tilapia; Oreochromis niloticus tissue) and three negative controls, treated the same way (twice with each of two indexed primers, for a total of 16 replicates of positive controls and 12 replicates of negative controls). Using tissue-derived DNA as a positive control allowed us to assess non-amplifications as deriving from sample-specific, rather than PCR-condition-specific causes, and selecting a non-native species as the tissue source allowed us to identify putative cross-contamination among samples (all Tilapia sequences should derive from the laboratory rather than the field). 150bp paired-end sequencing was carried out on an Illumina Nextseq.

\section{Sequence Processing and Bioinformatics}

We processed the Nextseq reads with a custom Unix-based script (O'Donnell, 2015), which calls existing third-party scripts to move from raw sequence data to a quality-controlled dataset of operational taxonomic units (OTUs). See Supplementary Methods for further bioinformatics details.

\section{Contamination Removal and Sequencing-Depth Normalization}

We used a Bayesian site-occupancy modeling method to estimate the probability of the OTU representing a true positive detection (Ficetola et al., 2014; Lahoz-Monfort, Guillera-Arroita \& Tingley, 2015), fitting a binomial distribution to OTU occurrences across replicates of each environmental sample, and rarefied OTUs in each sample using the smallest number of reads we observed in a single sample (124,041 reads; (Gotelli \& Colwell, 2001)) to standardize estimates of taxon richness across samples. We generated 1000 rarefied datasets, and unless otherwise specified below, we report results from one representative rarefied dataset consisting of $11.8 \times 10^{\wedge} 6$ reads reads

157 representing 1664 unique OTUs. The results do not depend significantly on the choice of rarefaction

158 replicates; for example, replicates differed only trivially in OTU richness $($ mean $=1662, \mathrm{sd}=9.5)$

159 and did not show different spatial trends among replicates. For beta and gamma diversity measures,

160 in particular, OTU identity is of importance, and accordingly we show data derived from the entire

161 set of rarefaction replicates. Finally, for each water sample, we then averaged across the four PCR 
162 replicates to estimate the abundance of each OTU. The complete eDNA dataset and analytical scripts

163 are publicly available on Dryad (Accession: doi:10.5061/dryad.04tq4). See Supplementary

164 Methods for further sequence processing details.

165

166 Our results do not depend strongly on decontamination or normalization procedures. Analyses of raw

167 OTU data (with no decontamination or normalization), of only the most common 100 OTUs, and of

168 only the least-common 500 OTUs, all produce the same trends in the quality-controlled and

169 normalized data (Suppl. Fig. 1). Similarly, rarefaction replicates retain the same strong trends

170 observed in our representative single replicate (Suppl. Fig. 2).

171

172

\section{Taxonomic Annotation of eDNA Sequences}

173 We annotated the final set of OTU sequences using the command-line BLAST + software (Camacho et al., 2009), searching against the complete NCBI nucleotide database (as of 12 October, 2015), with word size $=7$ and up to 1000 hits per query sequence retained. Those with no hits at $\mathrm{e}=10^{\wedge}-13(<$ ca. $85 \%$ identity) or better were treated as unannotated. Conflicting sequence annotations were resolved using the last common ancestor algorithm implemented in MEGAN (Huson et al., 2011). Disagreement among hits for a given OTU (i.e., where a single OTU is an equally good match to $>1$ taxon) was generally resolved at the level of taxonomic Family (83.2\% of reads; Supp. Table 1).

\section{Data Analysis, Community Composition, and Diversity}

182 Although amplicon sequencing produces read counts that may contain valuable information about target species abundances (Evans et al., 2016; Port et al., 2016) it remains difficult to interpret the results of amplicon studies in the context of quantitative ecology because the precise relationship between amplicon abundance and taxon abundance remains unknown and likely varies among taxa (Evans et al., 2016). Accordingly, our analyses used presence/absence information derived from sequence count data.

To assess the appropriateness of the spatial scale scale of sampling, we apportioned the observed variance in ecological distance (Jaccard) among sites, among transects (within sites), and among PCR replicates using a PERMANOVA. We calculated alpha diversity (= richness, or "density", sensu (Gotelli \& Colwell, 2001)) at both the OTU level and at the level of taxonomic family, treating individual transects as replicates within a geographic site. We calculated beta diversity (sensu Whittaker 1960, a measure of faunal change) both among transects within sites and among sites (using transect means within sites to calculate the latter), focusing on OTUs because of the loss of resolution associated with incomplete taxonomic annotation. We used Raup-Crick dissimilarity (Chase et al., 2011) to ensure the observed beta diversity trends were not strictly dependent upon changes in alpha diversity. We then evaluated gamma diversity (richness across sites within a region) by generating an accumulation curve for three sets of sites: more-urban $(\mathrm{N}=4$ sites $)$, less-urban $(\mathrm{N}=$ 4 sites), and all sites $(\mathrm{N}=8)$. We sampled each set of sites (with replacement) 25 times at each step in the accumulation curve to capture the distribution of site-specific richness.

We evaluated the relationships between diversity metrics and urbanization using linear and generalized linear regression, as well as mixed-effects models. Our data were nested, with three transect samples per site, and with each site having a single imperviousness value. To avoid pseudoreplication among transects, we used site means for linear and generalized linear regressions. For the mixed-effects models, we considered imperviousness as a fixed covariate and both site pair

208 and site identity as a random intercept terms. 
209

210

211

212

213

214

215

216

217

218

219

220

221

222

223

224

225

226

227

228

229

230

231

232

233

234

235

236

237

238

239

240

241

242

243

244

245

246

247

248

249

250

251

252

253

254

255

To approximate life-history diversity, we organized all OTUs for which a Family-level annotation was possible and classified each according to the following natural history attributes: Category (epifauna, infauna, demersal, pelagic, terrestrial); Habitat (terrestrial, freshwater, intertidal, subtidal); and Mobility (motile, sessile) using available reference materials such as (Kozloff, 1983). In some cases, Families included species with a range of classifications (e.g., Cardiidae are a bivalve family which includes infaunal and epifaunal cockles found both intertidal and subtidal habitats, with a range of motility); in such cases the Family was listed as having both attributes. In all, there were 19 unique life-history niches that combinations of these attributes described (e.g., "Sessile Intertidal Epifauna", etc.; Suppl. Table 2). We used these classifications to assess trends in the richness of these life-history groups with respect to imperviousness, and in a principal components analysis to assess differences in faunas among sites.

Finally, we used logistic regression and binomial tests to identify particular taxa, OTUs, and lifehistory characteristics significantly associated with imperviousness. We conducted all analyses in $\mathrm{R}$ v3.2.2 (R Core Team, 2015).

\section{Results}

Our representative rarefied eDNA (16s mtDNA) dataset recovered 1664 operational taxonomic units (OTUs; mean of 1000 rarefaction replicates $=1662$ OTUs \pm 9.5 ) from a wide array of taxa characteristic of the Puget Sound estuarine environment, with 10 animal phyla represented across 27 Classes, 65 Orders, and 135 Families (Table 1). Detections included iconic groups such as Metacarcinus (i.e., Cancer) crabs, birds of prey (Accipitridae), and marine mammals (Delphinidae), with the bulk of unique OTUs reflecting molluscs (45.1\%), chordates $(20.2 \%)$, and arthropods (15.9\%). $92 \%$ of reads ( $70 \%$ of OTUs) could be annotated with high confidence $\left(\mathrm{e}<10^{\wedge}-32\right)$. These annotations included many animal taxa common to Puget Sound or the surrounding environment (Table 1; see Supplemental Table 1 for full Family-level annotations).

The total variance in community-level ecological distance was attributable to differences among sites (38.6\%), among transects within sites $(45.4 \%)$, or among PCR replicates of the same water samples (15.9\%; PERMANOVA with Jaccard distance, $\mathrm{p}<0.001,999$ permutations, using OTU presenceabsence data). These results are consistent with earlier work in nearshore habitats (Port et al., 2016), reflecting differences in eDNA profiles at spatial scales on the order of tens to hundreds of meters (here, between transects separated by ca. $50-100 \mathrm{~m}$ ) and limited variability due to PCR and sequencing processes. Ordination of OTU data shows transect samples largely, but not exclusively, clustering within geographic sites (Suppl. Fig. 3).

\section{OTU Diversity and Urbanization}

OTU richness increased significantly with upland imperviousness (Fig. 2). Family-level richness reflected the overall richness trends (Fig. 2). The results were highly robust to different decontamination or normalization procedures (Suppl. Figs. 1 and 2).

Our paired sampling design controlled for potentially confounding geographically associated differences among sites. We observe the same strong positive OTU richness correlation with imperviousness in all 4 site pairs (Fig. 2), evidence that some aspect of urbanization - rather than confounding spatial differences among site pairs-explains the observed pattern. A mixed-effects 
256

257

258

259

260

261

262

263

264

265

266

267

268

269

270

271

272

273

274

275

276

277

278

279

280

281

282

283

284

285

286

287

288

289

290

291

292

293

294

295

296

297

298

299

300

301

302

model showed that imperviousness had a positive effect on richness after accounting for pair and site identity $(\mathrm{p}=0.018)$.

We calculated beta diversity (faunal turnover) at two different hierarchical scales: between sites and among transects within sites. Consistent with the high level of heterogeneity we observed among transects within sites, between-site beta diversity was uniformly high and did not differ for more- or less-urban sites (Whittaker's beta (1960); Wilcoxon test, $\mathrm{p}=0.58$ ). Focusing on the individual transects, however, revealed a strong decrease in within-site beta diversity with urbanization across all four site pairs: communities became more homogeneous (transects within sites became more similar) as watershed imperviousness increased (Fig. 2). Whittaker's beta (Whittaker, 1960) decreased from a mean of 0.816 when imperviousness was less than $10 \%$ to a mean of 0.546 when imperviousness was greater than $25 \%\left(\mathrm{R}^{\wedge} 2=0.93, \mathrm{p} 8.4 \times 10^{\wedge}-5\right)$. Raup-Crick dissimilarity among transects showed a similar trend, indicating that the urbanization-associated trend in transect-totransect variation in eDNA composition was greater than expected due to changes in alpha diversity alone.

Consistent with the trend in richness, more-urban sites had consistently higher gamma diversity than less-urban sites, as reflected in the completely non-overlapping OTU accumulation curves in those sets of sites (Fig 2). In total, more-urban sites had 1295 unique OTUs in 116 Families, while lessurban sites had 790 OTUs from 80 Families, respectively.

\section{Life-History Diversity and Urbanization}

Assessing individual characteristics of habitat and mobility, taxa with differing natural history characteristics were differentially associated with urbanization. For example, OTU richness tripled with greater urbanization among sessile taxa $\left(\mathrm{p}=1.7 \times 10^{\wedge}-5\right)$, but motile taxa increased only nonsignificantly $(p=0.054)$. Similarly, OTU richness in intertidal $\left(p=7.5 \times 10^{\wedge}-6\right)$ and subtidal $(p=$ $\left.3 \times 10^{\wedge}-5\right)$ taxa increased with imperviousness, terrestrial taxa showed no such trend $(\mathrm{p}=0.16)$.

Community shifts among natural history types reflected richness changes by taxonomic groups. At both the OTU- and Family level, eDNA richness increased with urbanization, most notably among bivalves and gastropods (Suppl. Fig. 4). Family-level bivalve richness rose, for example, from an average of 5 Families (37 OTUs) at $<10 \%$ imperviousness to 7.4 Families (111 OTUs) at $>25 \%$ imperviousness (Poisson GLM with log-link, $\mathrm{p}<0.01$ at family level, $10^{\wedge}-16$ at OTU level). Other taxa showed a more gradual increase in richness with imperviousness (Suppl. Fig. 5), resulting in an overall increase in the number of taxonomic Families. No abundant Family declined with imperviousness.

Combining ecological characteristics into tri-variate life-history categories (e.g., "intertidal sessile epifauna") revealed 19 unique Family categories present. Life-history richness increased with urbanization (Suppl. Fig. 6; $\mathrm{R}^{\wedge} 2=0.74, \mathrm{p}=0.006$ ), from a mean of 12.5 life histories per site in lessurban sites to a mean of 14.7 in more-urban sites, due to the concomitant increase in taxon richness at more-urban sites. Normalizing by the number of Families present at each site reveals a strong decrease in occupied life-histories-per-taxon with urbanization, from a mean of 0.66 in less-urban sites to 0.47 in more-urban sites (although the trend is nonsignficant; Suppl. Fig. 6; $\mathrm{R}^{\wedge} 2=0.38, \mathrm{p}=$ 0.1 ). Ordination of the life histories results in identifiable sites and urbanization categories (Suppl. Fig. 7), similar to the ordination plot for OTUs. 
304 Beyond community measures, we identified 46 individual OTUs - again dominated by bivalves (33

305 OTUs from 5 families) - that were positively correlated ( $\mathrm{p}<0.01$; logistic regression) with upland

306 imperviousness. Gastropods (5; limpets), urchins or sand dollars (7; not classifiable to family level),

307 and one fish OTU comprised the remaining 13 OTUs. Conversely, a single OTU was negatively

308 correlated with imperviousness (a mytilid mussel OTU). Providing some direct indication of human

309 influence on the nearshore Puget Sound, human OTU richness increased significantly with

310 imperviousness $(\mathrm{p}=0.01$; Poisson GLM), as did richness in selected taxa cultivated commercially

311 (Panopea, $\mathrm{p}=5 \times 10^{\wedge}-4 ;$ Bos, $\left.\mathrm{p}=0.005\right)$ or introduced taxa $\left(\right.$ Mya, $\left.\mathrm{p}=5.9 \times 10^{\wedge}-6\right)$.

312

313

Discussion

314 All organisms leave behind residual genetic signatures in their environments, which provide the

315 opportunity to explore patterns of diversity and community structure that may not be possible

316 otherwise. Here, we recovered these signatures from nearshore estuarine habitats along an urban

317 gradient, revealing strong trends in the diversity of animals and ecological roles present. While alpha

318 (site richness) and gamma (regional richness) diversity strongly increased with upland urbanization,

319 more-urban sites were significantly more homogeneous (within sites) than less-urban sites. Life-

320 history diversity largely paralleled these same trends, with a greater richness of ecological life

321 histories among taxa found in more urban areas, but greater redundancy in life-history niches among

322

323

324

325

326

327

328

329

330

331

332

333

334

335

336

337

338

339

340

341

342

343

344

345

346

347

348 these taxa. Taken together, our results suggest that more urbanized upland areas support larger suites of species, with less compositional variation, in and around downstream eelgrass habitats. Further, we find evidence that the mechanisms of land-sea interaction act at watershed scales, rather than at the larger scale of Puget Sound. These results also substantiate the idea that eDNA can be a powerful addition to traditional means of assessing human-ecosystem interactions.

\section{Trends in Diversity and Ecological Function with Urbanization}

Although dense urban areas do not necessarily decrease biodiversity in general (Ives et al., 2016) and the effects of urbanization on species richness appear to be taxon- and spatial-scale-specific (Shochat et al., 2006), the positive richness trend we see in Puget Sound 16s eDNA is nevertheless striking. Several plausible mechanisms could explain the increase in 16s eDNA richness, although our study design prevents us from assessing causation explicitly.

One likely explanation for the trend is the interaction between fauna sampled with eDNA and the kinds of habitat that are more common near urban settlements. Our study design attempted to sample identical habitats across all sites, however, there may be unobserved differences in habitats. For example, our results may reflect an increase in availability of muddy habitats associated with urbanization, and a concomitant increase in richness within those habitat patches.

A second plausible mechanism is that greater anthropogenic nutrient inputs into urban areas yields greater productivity. Urbanization greatly increases total nitrogen fluxes into rivers and estuaries (Rabalais et al., 2009; Mohamedali et al., 2011), and increased primary productivity, which may result from such fertilization, is generally_but not strictly-associated with increased secondary productivity (Leslie et al., 2005) and taxonomic diversity (Mittelbach et al., 2001; Whittaker \& Heegaard, 2003). However, Puget Sound, like many coastal systems, is dominated by marine derived nutrients (Mackas \& Harrison, 1997; Mohamedali et al., 2011), suggesting that any fertilization effect from small watersheds such as those we focus on here is unimportant. Each of the urban sites we 
350 sampled also has a wastewater treatment facility in the vicinity. However, all outflows from

351 treatment facilities occur in deep water offshore, far from our sampling areas, making any effect of

352 fertilization indirect at best. Wastewater treatment facilities could also increase richness by

353

354

355

356

357

358

359 concentrating genetic material originating elsewhere. However, although the increase in human OTUs we observe is consistent with this hypothesis, the great majority of DNA recovered stems from Puget Sound species rather than taxa likely to be dominant in human waste streams and none of our results is driven by exogenous eDNA.

Intriguingly, as eDNA communities increased in richness with urbanization, they also became more homogeneous. Others have found that increased subtidal sedimentation - associated with the kind of low-energy environments we sampled here - tended to make rocky reef communities more similar to one another (Balata, Piazzi \& Benedetti-Cecchi, 2007), and nutrient enrichment can have the same effect in lakes (Donohue et al., 2009). Our results are consistent with the idea that urbanization tends to homogenize communities even though the total number of unique taxa may increase (Urban et al., 2006; Piazzi \& Balata, 2008). A similar effect is also associated with non-indigenous species introductions (Rosenzweig, 2001), but non-indigenous species do not drive the trends we observe here. Although a comprehensive list of native taxa is not available against which to compare our results, the annotated Families are nearly all familiar native taxa from Puget Sound; moreover, the trends we report are consistent across even small subsets of the data (Suppl. Figs $1 \&$ 2), indicating our results do not depend upon a small set of potentially non-indigenous taxa.

More generally, beta diversity can help disentangle the ecological forces behind community assembly(Condit et al., 2002; Tuomisto, Ruokolainen \& Yli-Halla, 2003; Dornelas, Connolly \& Hughes, 2006; Chase, 2007, 2010; Chase \& Myers, 2011), by distinguishing niche-related deterministic processes from stochastic ones. Our observations are consistent with the idea that that deterministic, possibly niche-related, processes significantly influence Puget Sound nearshore communities: transect-to-transect beta-diversity declined steadily with an environmental gradient of urbanization independent of geographic space, and per-taxon life-history richness similarly declined (albeit nonsignificantly) across this same environmental gradient.

We expect different ecological patterns to be apparent at different spatial scales, and conversely, the scales of ecological patterns provide hints about the mechanisms driving those patterns (Levin, 1992). Given the site- and transect-level differences we observed, it seems likely that the mechanisms mediating the human-ecosystem interactions in Puget Sound occur at the watershed scale ( 100s of meters), rather than at larger scales of urbanization (e.g., Puget Sound scale, 10s of km). Urbanization does not appear to homogenize communities across sites; more-urban sites were just as different from

394 Regardless of the precise mechanism, the eDNA data reveal a strong signal of land-sea interaction

395 (Samhouri \& Levin, 2012). Especially in light of ever-increasing human population density in coastal 
396

397

398

399

400

401

402

403

404

405

406

407

408

409

410

411

412

413

414

415

416

417

418

419

420

421

422

423

424

425

426

427

428

429

430

431

432

433

434

435

436

437

438

439

440

441

442

areas worldwide (Neumann et al., 2015), our results suggest that eDNA can be a powerful tool for uncovering human-ecosystem interactions that might otherwise remain hidden.

\section{eDNA as an Emerging Tool for Ecological Analysis: Scale and Selectivity}

Ecology and related disciplines depend upon techniques to sample and describe communities, ecosystems, and their properties. However, any one set of samples yields a necessarily biased view of the world; ten different sampling methods can yield ten different results even with small numbers of target taxa (Valentini et al., 2015). This selectivity is usually intentional-e.g., settlement plates are designed to sample bryozoans rather than seals - but where unintentional, such selectivity can bias results in ways that often remain unexplored (Baker et al., 2016).

The rise of eDNA sampling has led to studies comparing molecular techniques either to traditional methods or to known communities. Single-taxon qPCR studies have compared favorably with traditional surveys in terms of detection rates (Jerde et al., 2011; Takahara et al., 2012; Eichmiller, Bajer \& Sorensen, 2014a; Laramie, Pilliod \& Goldberg, 2015), with sequence-based (i.e., metabarcoding) analyses proving more difficult to interpret relative to traditional sampling, in part because of difficulty of comparing detection rates across methods (Cowart et al., 2015). eDNA is an in-depth sampling technique that yields interesting and repeatable results; however, the absence of eDNA detection does not imply absence of taxon of interest (Roussel et al., 2015). One eDNA locus, or even several loci, will not reveal all of the taxa present in an area. Indeed, eDNA sampling with a different genetic locus — or even a different set of primers at the same locus — would have yielded a different suite of taxa (e.g., (Cowart et al., 2015)).

Consistent with earlier observations from a study of Zostera communities (Cowart et al., 2015), our single eDNA locus failed to detect epifauna known from the sampled sites. Hippolytid and crangonid shrimp, littorinid snails, idoteid isopods, and others were common in the field but absent from the eDNA (Samhouri et al.), likely due to amplification bias and primer mismatches. Such performance does not make eDNA inappropriate for biodiversity monitoring, but rather put sequenced-based sampling in the company of every other sampling technique (Shelton et al., 2016). Because the "true" community remains unknown (Shelton et al., 2016), it is impossible to evaluate error rate in an absolute sense for any field-based method. Given that nearly all $(>99 \%)$ of the taxa we detect here are known from local waters or the surrounding area, our false positive rate for eDNA appears to be very low. We suggest that community-level eDNA surveys be viewed in a light appropriate to any new sampling technique: biased relative to some unknown true value, but significantly complementing existing imperfect sampling techniques such as tow nets and other manual collections.

Finally, our results suggest that eDNA recovers fine-scale differences in ecological communities, such that transects tens of meters apart can be as different as transects kilometres apart. Nearly half $(45 \%)$ of the variance in ecological distance was due to differences between transects at the same sampling site, consistent with the fine-grained spatial resolution reported by (Port et al., 2016) in another nearshore eDNA amplicon study. This observation supports a growing sense that eDNA may travel only limited distances away from its sources, depending upon the environmental context (Eichmiller, Bajer \& Sorensen, 2014a; Deiner \& Altermatt, 2014; Laramie, Pilliod \& Goldberg, 2015), and provide further evidence that eDNA variation at small spatial scales is more likely signal than noise. Nevertheless, it is not obvious why eDNA might exhibit such variability on the order of 
443 tens of meters (in this study and in others; (Eichmiller, Bajer \& Sorensen, 2014b; Port et al., 2016)), 444 but simultaneously feature the genetic signatures of species that are not in the immediate vicinity. 445 Examples here include terrestrial and aquatic taxa, whose DNA must have travelled at least some 446 distance into the intertidal habitats sampled. One explanation is that - if genetic material is detectable

447

448

449

450

451

452

453

454

455

456

457

458

459

460

461

462

463

464

465

466

467

468

469

470

471

472

473

474

475

476

477

478

479

480

481

482

483

484

485

486

487

488

489

490

as a steady-state balance of generation, degradation, advection, and diffusion away from a point source - such transportation is to be expected at low levels, even when the bulk of genetic material remains close to its source. Consistent with this model, the great majority of taxa in our data are marine, with non-marine taxa only at low levels ( $6 \%$ of reads including human DNA; $3 \%$ not including human DNA; see Suppl. Table 1).

\title{
Conclusion
}

Sampling using eDNA sequencing offers a breadth of taxonomic coverage valuable for both basic and applied ecology. Our results demonstrate the power of this technique for assessing humanecosystem interactions in a nearshore environment, revealing significant trends in animal diversity and life history likely linked to human alteration of upland habitats. Like all sampling methods, eDNA offers a view of the world that is both biased and incomplete, in the sense that surveys using a given gene will detect some taxa and not others. Traditional sampling has analogous drawbacks. Here, data from a single genetic locus provided a reasonably holistic view of the Puget Sound nearshore ecosystem - encompassing taxa as diverse as high-intertidal barnacles, birds of prey, and subtidal bivalves, from a wide variety of ecologically-linked habitats - that strongly suggests urbanization has generated unexpected consequences for a large number of nearshore taxa, particularly those with sessile lifestyles. Consistent with (Samhouri et al.; Blake, Duffy \& Richardson, 2014; Ives et al., 2016), we see these results as a counterexample to the idea that humans uniformly decrease biodiversity. Rather, the observation that more urbanized areas support larger, but more homogeneous, suites of species indicates a more nuanced effect of human alteration on nearshore communities.

\section{Additional Information}

\author{
Acknowledgments \\ We thank J.Port, L. Sassoubre, and A. Boehm; A. Stier, and P. Levin; M. Dethier, E. Heery, J. Toft, and J. Cordell; R. \\ Morris and V. Armbrust; J. Kralj; A. Wong, E. Garrison, J. Levy, M. Klein, and E. Buckner; coastal property owners for \\ access to field sites; and the Helen R. Whiteley Center at Friday Harbor Laboratorie, and two anonymous reviewers.

\section{Data Accessibility} \\ The article's supporting data, metadata, and analytical code can be accessed at Dryad (accession: \\ doi:10.5061/dryad.04tq4) and Genbank (accession number pending).
}

\section{Figure captions}

FIGURE1 
491 Study site sampling locations and associated stream basins. Matched site pairs share a stream basin color.

492 More urban sites are open boxes, less urban are black boxes. Two-letter codes correspond to site names in

493

494

495

496

497

498

499

500

501

502

503

504

505

506

507

508

509

510

511

512

513 the Methods. Brown shading indicates areas with greater than $50 \%$ imperviousness.

\section{FIGURE 2}

Top Row: Rarefied OTU richness and imperviousness-a proxy for urbanization-in Puget Sound. Analysis of a single focal rarefaction draw. Left : Rarefied 16s eDNA richness (solid trendline reflects OTUs; dashed trendline reflects taxonomic Families). Site means (larger circles) among transect-level data points (smaller circles). Family data shifted slightly for clarity. Right: The same data by site pair ( $\mathrm{N}=4$ pairs of more- and less-urban sites), means plotted. Red lines indicate significant trends, grey lines indicate non-significant trends. Legends correspond to 2-letter site codes in Fig. 1. Middle Row: Left: Mean among-transect (withinsite) Whittaker's beta diversity for each of 1000 rarefaction draws from the overall OTU dataset, rarefied to create comparable sample sizes $\left(\mathrm{N}=1.3 \times 10^{\wedge} 5\right.$ OTUs per transect). Linear regression on site means, $\mathrm{R}^{\wedge}=0.95, \mathrm{p}=3.38 \times 10^{\wedge}-5$. Right: Site means highlight the site-pair trends for single focal rarefaction draw. Bottom Row: Regional (gamma) diversity, in OTUs-per-site, as an accumulation curve. Boxplots show variance due to sampling each each set of sites (with replacement) 1000 times from a pool of 1000 rarefaction draws from the overall OTU dataset, rarefied to create comparable sample sizes $\left(\mathrm{N}=1.3 \times 10^{\wedge} 5\right.$ OTUs per transect). Best-fit logarithmic curves shown for more-urban sites $(\mathrm{N}=4)$, less-urban sites $(\mathrm{N}=4)$, and all sites $(\mathrm{N}=8)$.

515

516

517

518

519

520

521

522

523

524

525

526

527

528

529

530

531

532

533

534

535

536

537

538

539

540

541

542

543

544

545
Baker DGL., Eddy TD., Mclver R., Schmidt AL., Thériault M-H., Boudreau M., Courtenay SC., Lotze HK. 2016. Comparative analysis of different survey methods for monitoring fish assemblages in coastal habitats. PeerJ 4:e1832. DOI: 10.7717/peerj.1832.

Balata D., Piazzi L., Benedetti-Cecchi L. 2007. Sediment disturbance and loss of beta diversity on subtidal rocky reefs. Ecology 88:2455-2461.

Blake RE., Duffy JE., Richardson JP. 2014. Patterns of seagrass community response to local shoreline development. Estuaries and Coasts 37:1549-1561.

Bright EA., Coleman PR., Rose AN., Urban ML. 2012. LandScan 2011. Digital dataset, Oakridge National Laboratory, Oakridge, TN, USA, web. ornl. gov/sci/landscan/index. shtml.

Camacho C., Coulouris G., Avagyan V., Ma N., Papadopoulos J., Bealer K., Madden TL. 2009. BLAST+: architecture and applications. BMC Bioinformatics 10:421.

Chase JM. 2007. Drought mediates the importance of stochastic community assembly. Proceedings of the National Academy of Sciences of the United States of America 104:17430-17434. DOI: 10.1073/pnas.0704350104.

Chase JM. 2010. Stochastic community assembly causes higher biodiversity in more productive environments. Science (New York, N.Y.) 328:1388-1391. DOI: 10.1126/science.1187820.

Chase JM., Kraft NJB., Smith KG., Vellend M., Inouye BD. 2011. Using null models to disentangle variation in community dissimilarity from variation in a-diversity. Ecosphere 2:art24.

Chase JM., Myers JA. 2011. Disentangling the importance of ecological niches from stochastic processes across scales. Philosophical transactions of the Royal Society of London. Series B, Biological sciences 366:2351-2363. DOI: 10.1098/rstb.2011.0063.

Condit R., Pitman N., Leigh EG., Chave J., Terborgh J., Foster RB., Núñez P., Aguilar S., Valencia R., Villa G., Muller-Landau HC., Losos E., Hubbell SP. 2002. Beta-diversity in tropical forest trees. Science (New York, N.Y.) 295:666-669. DOI: 10.1126/science.1066854.

Cowart DA., Pinheiro M., Mouchel O., Maguer M., Grall J., Miné J., Arnaud-Haond S. 2015. Metabarcoding Is Powerful yet Still Blind: A Comparative Analysis of Morphological and Molecular Surveys of Seagrass Communities. PloS One 10:e0117562.

Deiner K., Altermatt F. 2014. Transport Distance of Invertebrate Environmental DNA in a Natural River. PLoS One 9:e88786. DOI: 10.1371/journal.pone.0088786.

Dethier MN. 2010. Variation in recruitment does not drive the cline in diversity along an estuarine 
546

547

548

549

550

551

552

553

554

555

556

557

558

559

560

561

562

563

564

565

566

567

568

569

570

571

572

573

574

575

576

577

578

579

580

581

582

583

584

585

586

587

588

589

590

591

592

593

594

595

596

597

598

599

600

601

gradient. Marine Ecology Progress Series 410:43-54.

Donohue I., Jackson AL., Pusch MT., Irvine K. 2009. Nutrient enrichment homogenizes lake benthic assemblages at local and regional scales. Ecology 90:3470-3477.

Dornelas M., Connolly SR., Hughes TP. 2006. Coral reef diversity refutes the neutral theory of biodiversity. Nature 440:80-82. DOI: 10.1038/nature04534.

Eichmiller JJ., Bajer PG., Sorensen PW. 2014a. The Relationship between the Distribution of Common Carp and Their Environmental DNA in a Small Lake. PloS One 9:e112611.

Eichmiller JJ., Bajer PG., Sorensen PW. 2014b. The relationship between the distribution of common carp and their environmental DNA in a small lake. PloS one 9:e112611. DOI: 10.1371/journal.pone.0112611.

Evans NT., Olds BP., Turner CR., Renshaw MA., Li Y., Jerde CL., Mahon AR., Pfrender ME., Lamberti GA., Lodge DM. 2016. Quantification of mesocosm fish and amphibian species diversity via eDNA metabarcoding. Molecular Ecology Resources 16:25-41.

Ficetola GF., Pansu J., Bonin A., Coissac E., Giguet-Covex C., De Barba M., Gielly L., Lopes CM., Boyer F., Pompanon F., Others. 2014. Replication levels, false presences and the estimation of the presence/absence from eDNA metabarcoding data. Molecular Ecology Resources 15:543-556.

Fry JA., Xian G., Jin S., Dewitz JA., Homer CG., LIMIN Y., Barnes CA., Herold ND., Wickham JD. 2011. Completion of the 2006 national land cover database for the conterminous United States. Photogrammetric engineering and remote sensing 77:858-864.

Gotelli NJ., Colwell RK. 2001. Quantifying biodiversity: procedures and pitfalls in the measurement and comparison of species richness. Ecology Letters 4:379-391.

Heerhartz SM., Dethier MN., Toft JD., Cordell JR., Ogston AS. 2014. Effects of shoreline armoring on beach wrack subsidies to the nearshore ecotone in an estuarine fjord. Estuaries and Coasts 37:1256-1268.

Huson DH., Mitra S., Ruscheweyh H-J., Weber N., Schuster SC. 2011. Integrative analysis of environmental sequences using MEGAN4. Genome research 21:1552-1560.

Ives CD., Lentini PE., Threlfall CG., Ikin K., Shanahan DF., Garrard GE., Bekessy SA., Fuller RA., Mumaw L., Rayner L., Others. 2016. Cities are hotspots for threatened species. Global Ecology and Biogeography 25:117-126.

Jerde CL., Mahon AR., Chadderton WL., Lodge DM. 2011. "Sight-unseen" detection of rare aquatic species using environmental DNA. Conservation Letters 4:150-157.

Karr JR. 1981. Assessment of biotic integrity using fish communities. Fisheries 6:21-27.

Kozloff EN. 1983. Seashore life of the northern Pacific coast: an illustrated guide to northern California, Oregon, Washington, and British Columbia. University of Washington Press Seattle.

Lahoz-Monfort JJ., Guillera-Arroita G., Tingley R. 2015. Statistical approaches to account for false positive errors in environmental DNA samples. Molecular Ecology Resources.

Laramie MB., Pilliod DS., Goldberg CS. 2015. Characterizing the distribution of an endangered salmonid using environmental DNA analysis. Biological Conservation 183:29-37.

Leslie HM., Breck EN., Chan F., Lubchenco J., Menge BA. 2005. Barnacle reproductive hotspots linked to nearshore ocean conditions. Proceedings of the National Academy of Sciences of the United States of America 102:10534-10539.

Levin SA. 1992. The problem of pattern and scale in ecology. Ecology 73.

Mackas DL., Harrison PJ. 1997. Nitrogenous Nutrient Sources and Sinks in the Juan de Fuca Strait/Strait of Georgia/Puget Sound Estuarine System: Assessing the Potential for Eutrophication. Estuarine, Coastal and Shelf Science 44:1-21. DOI: 10.1006/ecss.1996.0110.

Minnesota Population Center. 2011. National historical geographic information system: Version 2.0. Minneapolis, MN: University of Minnesota.

Mittelbach GG., Steiner CF., Scheiner SM., Gross KL., Reynolds HL., Waide RB., Willig MR., Dodson SI., Gough L. 2001. What is the observed relationship between species richness and productivity? Ecology 82:2381-2396.

Mohamedali T., Roberts M., Sackmann B., Kolosseus A. 2011. Puget Sound Dissolved Oxygen Model Nutrient Load Summary for 1999-2008.

Neumann B., Vafeidis AT., Zimmermann J., Nicholls RJ. 2015. Future coastal population growth and exposure to sea-level rise and coastal flooding--a global assessment. PloS one 10:e0118571. DOI: 10.1371/journal.pone.0118571.

Niemi GJ., McDonald ME. 2004. Application of ecological indicators. Annual Review of Ecology, 
602

603

604

605

606

607

608

609

610

611

612

613

614

615

616

617

618

619

620

621

622

623

624

625

626

627

628

629

630

631

632

633

634

635

636

637

638

639

640

641

642

643

644

645

646

647

648

649

650

651

652

653

654

655

656

657

Evolution, and Systematics:89-111.

NOAA. 2013. NOAA's Coastal Change Analysis Program (C-CAP) 2006 Regional Land Cover Data Coastal United States. National Ocean Service (NOS), Office for Coastal Management (OCM).

O'Donnell JL. 2015.banzai. Available at https://github.com/jimmyodonnell/banzai

O’Donnell James L., Kelly Ryan P., Lowell Natalie., Port JA. 2016. Indexed PCR Primers Induce Template-Specific Bias In Large-Scale DNA Sequencing Studies. PLoSOne in press.

OpenStreetMap. 2013. OpenStreetMap - Washington State Roads, OpenStreetMap contributors.

Piazzi L., Balata D. 2008. The spread of Caulerpa racemosa var. cylindracea in the Mediterranean Sea: an example of how biological invasions can influence beta diversity. Marine Environmental Research 65:50-61.

Port JA., O'Donnell JL., Lowell N., Romero-Maraccini O., Kelly RP. 2016. Assessing the Vertebrate Community of a Kelp Forest Ecosystem Using Environmental DNA. Molecular Ecology 25:527-541.

Puget Sound Nearshore Ecosystem Restoration Project. 2010. Puget Sound Basin PSNERP Database.

Puget Sound Partnership. 2011. Leadership Council Resolution 2011-01: Adopting an Ecosystem Recovery Target for Eelgrass.

R Core Team. 2015. R: A Language and Environment for Statistical Computing.

Rabalais NN., Turner RE., Diaz RJ., Justic D. 2009. Global change and eutrophication of coastal waters. ICES Journal of Marine Science 66:1528-1537. DOI: 10.1093/icesjms/fsp047.

Renshaw MA., Olds BP., Jerde CL., McVeigh MM., Lodge DM. 2015. The room temperature preservation of filtered environmental DNA samples and assimilation into a phenol--chloroform--isoamyl alcohol DNA extraction. Molecular Ecology Resources 15:168-176.

Riaz T., Shehzad W., Viari A., Pompanon F., Taberlet P., Coissac E. 2011. ecoPrimers: inference of new DNA barcode markers from whole genome sequence analysis. Nucleic Acids Research 39:e145e145.

Rice JC., Rochet M-J. 2005. A framework for selecting a suite of indicators for fisheries management. ICES Journal of Marine Science 62:516-527.

Rosenzweig ML. 2001. The four questions: What does the introduction of exotic species do to diversity? Evolutionary Ecology Research 3:361-367.

Roussel J-M., Paillisson J-M., Treguier A., Petit E. 2015. The downside of eDNA as a survey tool in water bodies. Journal of Applied Ecology 52:823-826.

Samhouri JF., Shelton AO., Williams GD., Feist B., Hennessey S., Bartz K., O'Donnell JL., Sheer M., Levin PS. How much city is too much city? Biodiversity and ecosystem functions along an urban gradient at the land-sea interface in Puget Sound. Journal of Applied Ecology In Revision.

Samhouri JF., Levin PS. 2012. Linking land-and sea-based activities to risk in coastal ecosystems. Biological Conservation 145:118-129.

Scyphers SB., Picou JS., Powers SP. 2015. Participatory Conservation of Coastal Habitats: The Importance of Understanding Homeowner Decision Making to Mitigate Cascading Shoreline Degradation. Conservation Letters 8:41-49. DOI: 10.1111/conl.12114.

Shelton AO., O'Donnell JL., Samhouri JF., Lowell N., Williams GD., Kelly RP. 2016. A framework for inferring biological communities from environmental DNA. Ecological Applications in press.

Shochat E., Warren PS., Faeth SH., McIntyre NE., Hope D. 2006. From patterns to emerging processes in mechanistic urban ecology. Trends in ecology \& evolution 21:186-191.

Takahara T., Minamoto T., Yamanaka H., Doi H., Kawabata Z. 2012. Estimation of fish biomass using environmental DNA. PloS One 7:e35868.

Thomsen PF., Kielgast J., Iversen LL., Møller PR., Rasmussen M., Willerslev E. 2012. Detection of a diverse marine fish fauna using environmental DNA from seawater samples. PLoS One 7:e41732.

Thomsen PF., Willerslev E. 2015. Environmental DNA--an emerging tool in conservation for monitoring past and present biodiversity. Biological Conservation 183:4-18.

Tuomisto H., Ruokolainen K., Yli-Halla M. 2003. Dispersal, environment, and floristic variation of western Amazonian forests. Science (New York, N.Y.) 299:241-244. DOI: 10.1126/science.1078037.

Turner CR., Barnes MA., Xu CCY., Jones SE., Jerde CL., Lodge DM. 2014. Particle size distribution and optimal capture of aqueous macrobial eDNA. Methods in Ecology and Evolution 5:676-684.

Tyson GW., Chapman J., Hugenholtz P., Allen EE., Ram RJ., Richardson PM., Solovyev V V., Rubin EM., Rokhsar DS., Banfield JF. 2004. Community structure and metabolism through reconstruction of microbial genomes from the environment. Nature 428:37-43.

Urban MC., Skelly DK., Burchsted D., Price W., Lowry S. 2006. Stream communities across a rural-- 
658

659

660

661

662

663

664

665

666

667

668

669

670

671

672

673

674

675

676

urban landscape gradient. Diversity and Distributions 12:337-350.

US Army Corps of Engineers. 2012. User's Guide For Nationwide Permits in Washington State.

Valentini A., Taberlet P., Miaud C., Civade R., Herder J., Thomsen PF., Bellemain E., Besnard A., Coissac E., Boyer F., Others. 2015. Next-generation monitoring of aquatic biodiversity using environmental DNA metabarcoding. Molecular ecology.

Venter JC., Remington K., Heidelberg JF., Halpern AL., Rusch D., Eisen JA., Wu D., Paulsen I., Nelson KE., Nelson W. 2004. Environmental genome shotgun sequencing of the Sargasso Sea. Science 304:66-74.

Weisberg SB., Ranasinghe JA., Dauer DM., Schaffner LC., Diaz RJ., Frithsen JB. 1997. An estuarine benthic index of biotic integrity (B-IBI) for Chesapeake Bay. Estuaries 20:149-158.

Whittaker RH. 1960. Vegetation of the Siskiyou mountains, Oregon and California. Ecological Monographs 30:279-338.

Whittaker RJ., Heegaard E. 2003. What is the observed relationship between species richness and productivity? Comment. Ecology 84:3384-3390.

Yutin N., Suzuki MT., Teeling H., Weber M., Venter JC., Rusch DB., Béjà O. 2007. Assessing diversity and biogeography of aerobic anoxygenic phototrophic bacteria in surface waters of the Atlantic and Pacific Oceans using the Global Ocean Sampling expedition metagenomes. Environmental microbiology 9:1464-1475. 


\section{Figure 1}

Study site sampling locations and associated stream basins.

Study site sampling locations and associated stream basins. Matched site pairs share a stream basin color. More urban sites are open boxes, less urban are black boxes. Two-letter codes correspond to site names in the Methods. Brown shading indicates areas with greater than $50 \%$ imperviousness. 


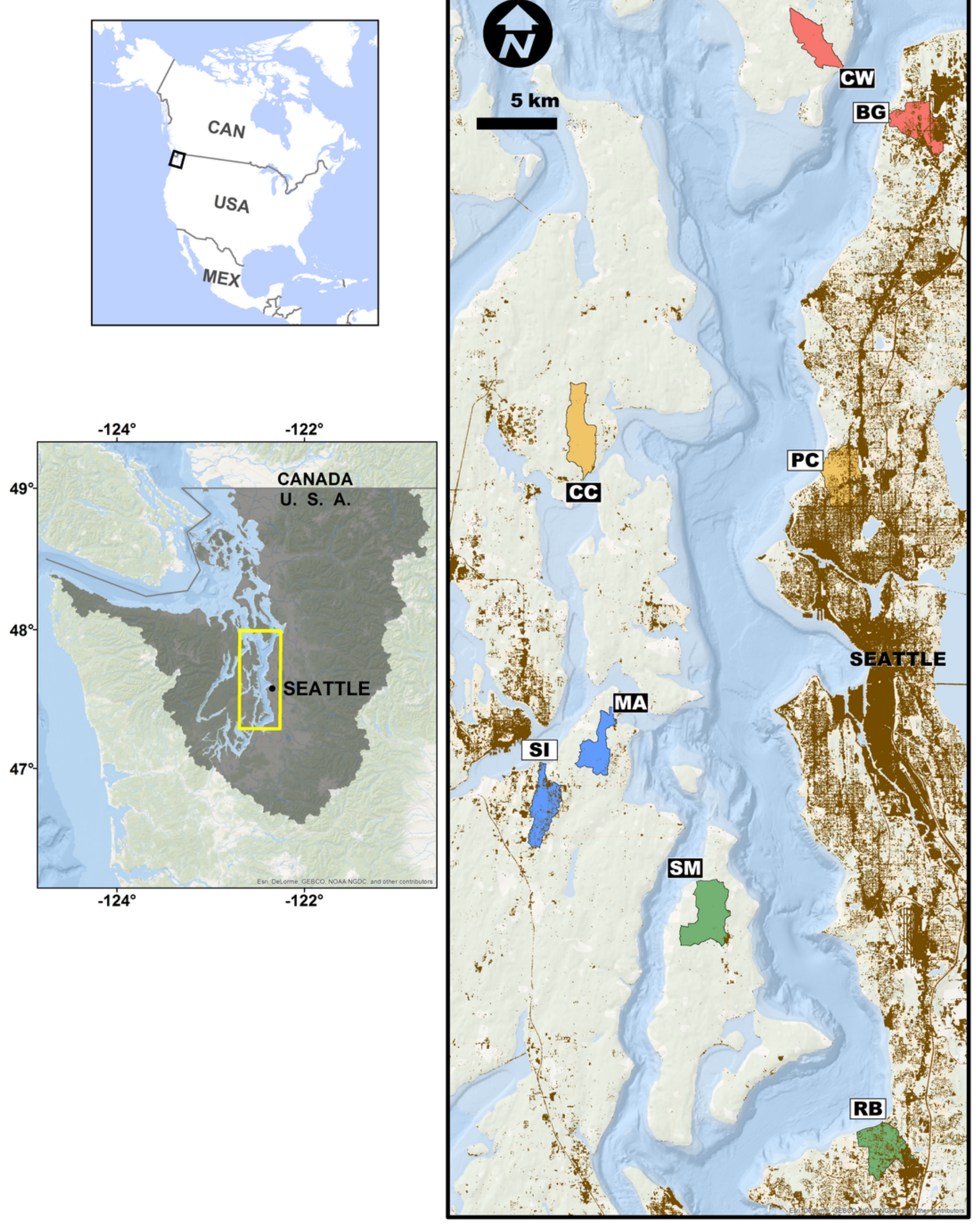




\section{Figure 2 (on next page)}

Alpha, beta, and gamma diversity recovered from water samples in Puget Sound along an urbanization gradient.

Top Row: Rarefied OTU richness and imperviousness-a proxy for urbanization-in Puget Sound. Analysis of a single focal rarefaction draw. Left : Rarefied 16s eDNA richness (solid trendline reflects OTUs; dashed trendline reflects taxonomic Families). Site means (larger circles) among transect-level data points (smaller circles). Family data shifted slightly for clarity. Right: The same data by site pair ( $\mathrm{N}=4$ pairs of more- and less-urban sites), means plotted. Red lines indicate significant trends, grey lines indicate non-significant trends.

Legends correspond to 2-letter site codes in Fig. 1. Middle Row: Left: Mean among-transect (within-site) Whittaker's beta diversity for each of 1000 rarefaction draws from the overall OTU dataset, rarefied to create comparable sample sizes ( $N=1.3 \times 10^{\wedge} 5$ OTUs per transect). Linear regression on site means, $R^{\wedge}=0.95, p=3.38 \times 10^{\wedge}-5$. Right: Site means highlight the site-pair trends for single focal rarefaction draw. Bottom Row: Regional (gamma) diversity, in OTUs-per-site, as an accumulation curve. Boxplots show variance due to sampling each each set of sites (with replacement) 1000 times from a pool of 1000 rarefaction draws from the overall OTU dataset, rarefied to create comparable sample sizes $\left(\mathrm{N}=1.3 \times 10^{\wedge} 5\right.$ OTUs per transect). Best-fit logarithmic curves shown for more-urban sites $(N=4)$, less-urban sites $(\mathrm{N}=4)$, and all sites $(\mathrm{N}=8)$. 

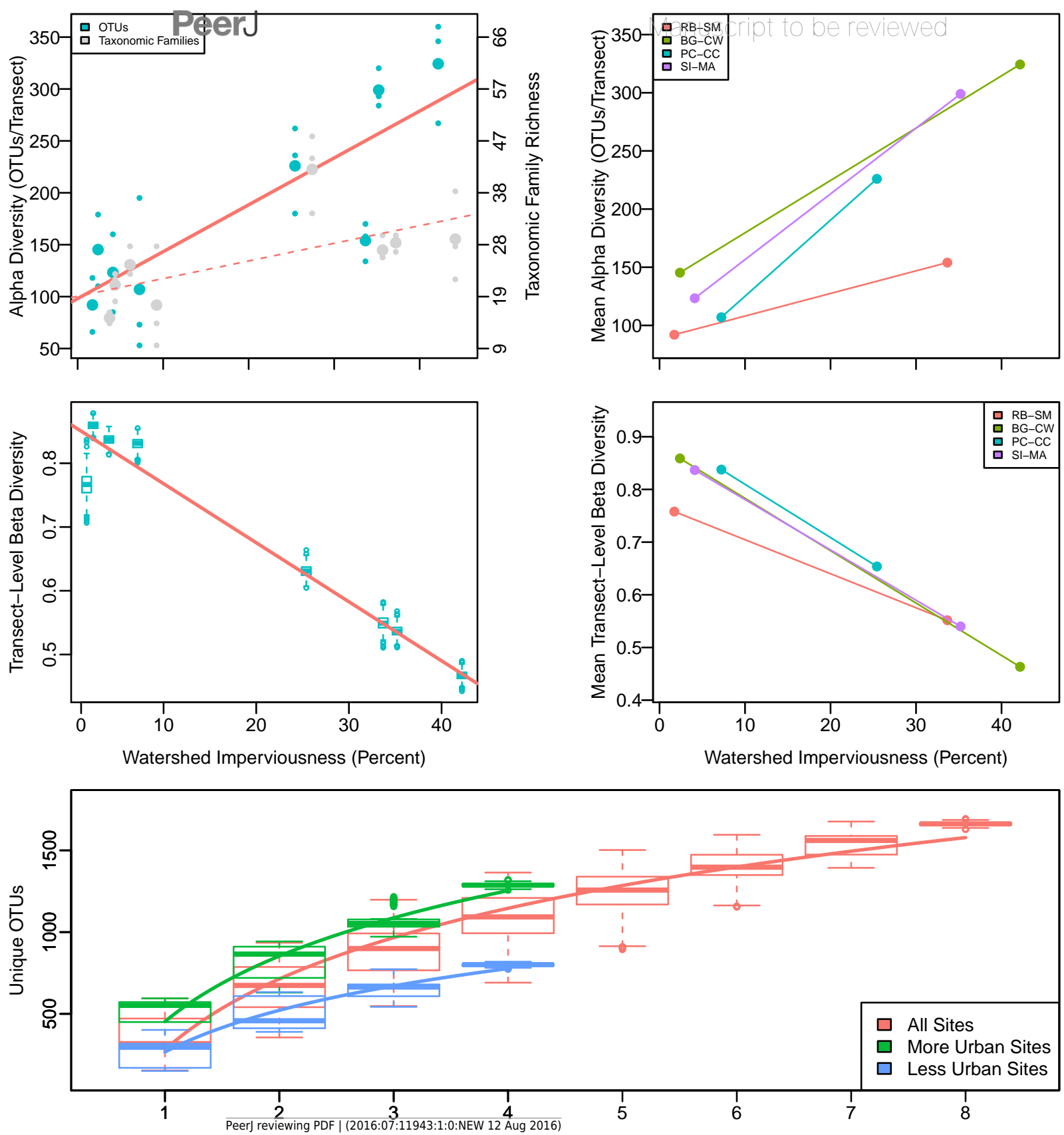

N Sites 


\section{Table $\mathbf{1}$ (on next page)}

Summary of 16s Read Annotations

Summary of taxonomic annotations for $16 \mathrm{~S}$ reads; for full annotations, see Supplementary Material. 
1 Tables

\begin{tabular}{|c|c|c|c|c|}
\hline PHYLUM & $\begin{array}{l}\text { CLASSE } \\
\mathrm{S}\end{array}$ & ORDERS & FAMILIES & OTHER RANK \\
\hline MOLLUSCA & 3 & 6 & 34 & 9 \\
\hline ARTHROPODA & 6 & 13 & 29 & 10 \\
\hline CHORDATA & 5 & 28 & 37 & 28 \\
\hline BRYOZOA & 1 & 2 & 10 & 2 \\
\hline ECHINODERMATA & 5 & 8 & 13 & 4 \\
\hline NEMERTEA & 3 & 2 & 8 & 0 \\
\hline HEMICHORDATA & 1 & 1 & 1 & 0 \\
\hline ENTOPROCTA & 1 & 1 & 1 & 0 \\
\hline PORIFERA & 1 & 2 & 2 & 0 \\
\hline
\end{tabular}

2

3 TABLE1

4 Summary of taxonomic annotations for $16 \mathrm{~S}$ reads; for full annotations, see Supplementary Material. 